\title{
Division of work
}

Among all chapters in the two volumes, the ones on preprocessing and files are written by Lei Feng. Xingni Zhou wrote the rest. Final compilation and editing was done by Qiguang Miao. 
